\title{
Coexistence of periods in a bisecting bifurcation
}

\author{
V. Botella-Soler ${ }^{\mathrm{a}, \mathrm{b}}$, J.A. Oteo ${ }^{\mathrm{a}}$, J. Ros ${ }^{\mathrm{a}, \mathrm{b}}$ \\ ${ }^{a}$ Departament de Física Teòrica, Universitat de València, 46100-Burjassot, València, \\ Spain \\ ${ }^{b}$ IFIC, Universitat de València-CSIC, 46100-Burjassot, València, Spain
}

\begin{abstract}
The inner structure of the attractor appearing when the Varley-GradwellHassell population model bifurcates from regular to chaotic behaviour is studied. By algebraic and geometric arguments the coexistence of a continuum of neutrally stable limit cycles with different periods in the attractor is explained.
\end{abstract}

Keywords: piecewise map, bifurcations, route to chaos

\section{Introduction}

Since the first discoveries of chaotic behavior in discrete maps, there has been great interest in the description of how the dynamics of a map changes from regular to chaotic, and viceversa, when a parameter of the map is varied. The so-called routes to chaos have been widely studied for smooth maps where well-known phenomena such as period-doubling, saddle-node bifurcations or intermittency appear [1, 2, 3]. The study of piecewise defined maps, which are useful in the mathematical description of mechanical systems with friction or collisions and of electrical circuits with switching components, has introduced new phenomenology [4, 5].

Here we consider a one-dimensional piecewise smooth discontinuous map originally proposed in studies on population ecology [6, 7], the Varley-GradwellHassell map (henceforth referred to as VGH map). It is a three-parameter map composed of a linear part and a power-law decreasing piece. It may present both regular and chaotic behavior, and for a critical value of one of its parameters the system undergoes an abrupt order-to-chaos transition. In [8] it was shown that at this transition point, the system has a continuum of neutrally stable limit cycles, i.e. cycles with multiplier unity. That situation 
appears also in two-dimensional maps and in a recent work [9] we have named such bifurcations bisecting bifurcations for a reason that will become clear in the following sections. The interesting characteristic of the bisecting bifurcation in the VGH map is that neutrally stable limit cycles with different periods coexist at the transition point. Their periods and their distribution in phase space depend on the location of the discontinuity of the map. Our previous results are here sharpened by invoking algebraic and geometric arguments.

The structure of the paper is as follows. In Section 2 we introduce the VGH map and its bisecting bifurcation. In Section 3 we explain a way to study algebraically the existence and location of this type of bifurcations. In Section 4, using the cobweb diagram of the map we provide a geometrical explanation of the bisecting bifurcation as well as the reason for the coexistence of different periods. In Section 5 we detail the structure of the attractor and of the basins of attraction at the bifurcation point. And finally, in Section 6 we discuss the main results presented in the paper.

\section{The VGH map}

The VGH map is defined in $[0, \infty)$ and reads

$$
V(x)= \begin{cases}r x, & x \leq c \\ r x^{1-b}, & x>c\end{cases}
$$

where $r, b>1$ and $c>0$. When $c=1$ the system is continuous, being discontinuous otherwise. The VGH map presents both regular and chaotic behavior and its dynamics exhibits a number of phenomena including interior crises and crisis-induced intermittency [8].

A customary construction of the bifurcation diagram by varying $b$, for fixed $r$ and $c$, shows an order-to-chaos bifurcation at $b=2$. For $b<2$ the system is regular with a single periodic attractor whose period depends on $c$. When $b>2$ the system is chaotic without further regular window. This is clear from the bifurcation diagrams in Figure 1 where three different values of the parameter $\mathrm{c}$ are considered. The top and bottom panels of the figure stand for cases where the map is discontinuous $(c=0.8,1.2)$ and the middle panel shows the bifurcation diagram in the continuous case $(c=1)$. At the bifurcation point $b=2$ all these diagrams exhibit a vertical segment which constitutes the focus of this work. 
Detailed numerical experiments, buttressed by the arguments in the next sections, demonstrate that any initial condition end up in a neutrally stable limit cycle. There is precisely an infinity of them which form the vertical segment in the diagrams of Figure 1. It is worthwhile to point out that the numerical detection of this continuum of attractors which mediates the bifurcation presents three main difficulties. First of all it exists only for the precise numerical value $b=2$. Then, an accurate enough machine-representation of the parameter values must be used in the sampling. Secondly, as the number of neutrally stable periodic orbits that exist for $b=2$ is infinite, a large enough number of initial conditions must be used to generate the vertical segment in the diagram. Finally, the whole attractor can easily be mistaken for a single chaotic attractor unless properly scrutinized. The algebraic and geometric analyses in the next sections are of great help in the correct identification of the vertical segment in the bifurcation diagram.

\section{Localization of the bifurcation in parameter space}

In this section we prove that for the VGH map the bisecting bifurcation takes place only at $b=2$. We start by introducing a change of variables that permits us to exactly linearize the VGH map

$$
z \equiv 2 \log (x) / \log (r), \quad \xi \equiv 2 \log (c) / \log (r)
$$

In terms of $z$ and $\xi$, the VGH map can be cast in the following linear form

$$
L(z)= \begin{cases}z+2, & z \leq \xi \\ (1-b) z+2, & z>\xi\end{cases}
$$

This linearized version allows the following algebraic approach. In the new form $L(z)$, the elements $\left\{z_{1}, z_{2}, \ldots, z_{T}\right\}$ of a cycle of period $T$ satisfy

$$
\begin{aligned}
& z_{n}=\left\{\begin{array}{ll}
z_{n-1}+2, & z_{n-1} \leq \xi, \\
(1-b) z_{n-1}+2 & z_{n-1}>\xi,
\end{array} \quad n=2, \ldots, T,\right. \\
& z_{1}= \begin{cases}z_{T}+2, & z_{T} \leq \xi, \\
(1-b) z_{T}+2, & z_{T}>\xi .\end{cases}
\end{aligned}
$$

These expressions can be cast in the form

$$
\begin{aligned}
z_{1}+\alpha_{T} z_{T} & =2 \\
z_{n}+\alpha_{n-1} z_{n-1} & =2, \quad n=2,3, \ldots, T
\end{aligned}
$$


with coefficients

$$
\alpha_{k}= \begin{cases}-1, & z_{k} \leq \xi \\ (b-1), & z_{k}>\xi\end{cases}
$$

The linear system of equations (5) can be expressed in matrix form

$$
\sum_{j=1}^{T} A_{i j} z_{j}=2, \quad i=1,2, \ldots, T,
$$

where the matrix $A$ is

$$
A=\left(\begin{array}{cccccc}
\alpha_{1} & 1 & 0 & \ldots & \ldots & 0 \\
0 & \alpha_{2} & 1 & 0 & \ldots & 0 \\
0 & 0 & \alpha_{3} & 1 & \ldots & 0 \\
\ldots & \ldots & \ldots & \ldots & \ldots & \ldots \\
0 & 0 & \ldots & \ldots & \alpha_{T-1} & 1 \\
1 & 0 & \ldots & \ldots & 0 & \alpha_{T}
\end{array}\right) .
$$

In order to find an attractor consisting of infinitely many limit cycles of period $T$ we need the system of equations (5) to be compatible and indeterminate. A necessary condition is therefore $\operatorname{det}(A)=0$. The determinant of the matrix $A$ is

$$
\operatorname{det}(A)=\prod_{k=1}^{T} \alpha_{k}+(-1)^{T+1} .
$$

If the cycle has $d$ of its elements satisfying $z_{i}>\xi$ this gives

$$
\operatorname{det}(A)=(-1)^{T-d}(b-1)^{d}+(-1)^{T+1},
$$

which vanishes for $b=0$ and, if $d$ is even, also for $b=2$. Since we are only interested in $b>1$, the only possible solution is then $b=2$. This is precisely the value found from the bifurcation diagrams in Section 2, It is important to notice that this value of $b$ and equation (3) imply neutral stability for any possible cycle. Of course, the argument in this section does not complete the analysis of the vertical segment in those diagrams. It only excludes any other value of $b$ as candidate. In the next Section we will explicitly show that the infinite set of periodic orbits actually exist. 


\section{Geometrical explanation of the bisecting bifurcation}

Next we develop an heuristic explanation for the emergence of a bisecting bifurcation based on the cobweb diagram of a general one-dimensional piecewise defined map $f(x ; p)$ with parameter $p$. Notice that an infinite set of regular trajectories of period $n$ will be observed for a critical value $p_{c}$ of the parameter when the $n$th iterate of the map $f^{[n]}$ has a piece which is co-linear with the bisectrix. Hence, the name we are using for these bifurcations. More explicitly, at the bifurcation, $f^{[n]}$ will be of the form

$$
f^{[n]}\left(x ; p_{c}\right)= \begin{cases}\cdots, & \cdots, \\ x, & x \in\left(x_{l}, x_{r}\right), \\ \cdots, & \cdots\end{cases}
$$

An instance of this phenomenon in the VGH map is given by its second iterate which reads

$$
V^{[2]}(x)= \begin{cases}r^{2} x, & x \leq \frac{c}{r} \\ r^{2-b} x^{1-b}, & \frac{c}{r}<x \leq c \\ r^{2-b} x^{(1-b)^{2}}, & c<x \leq\left(\frac{r}{c}\right)^{\frac{1}{b-1}} \\ r^{2} x^{1-b}, & x>c, x>\left(\frac{r}{c}\right)^{\frac{1}{b-1}}\end{cases}
$$

When $b=2$ the third piece becomes $x$ and we find a bisecting bifurcation mediated by an infinite set of neutrally stable period-2 limit cycles provided $c<\sqrt{r}$. This is illustrated in Figure 2 where three cobweb plots of $V^{[2]}(x)$ for values $b=1.6,2.0,2.4$ and $c=1$ are shown. In the three cases the same three initial conditions have been evaluated. When $b=1.6$, all trajectories converge to the stable fixed point. In the critical case, when $b=2$, each initial condition evolves to a different neutrally stable fixed point of $V^{[2]}(x)$. For $b=2.4$ the trajectories are chaotic.

In principle, several iterates of $f$ can have pieces co-linear with the bisectrix simultaneously for the same value of $p_{c}$. In such cases, infinite sets of cycles of different periods will coexist when $p=p_{c}$. This is the case for the VGH map. When $b=2$ and $c>1$ the neutrally stable limit cycles can show different periods. This is illustrated in Figure 3 where the evolution in the cobweb diagram and the trajectories of two different initial conditions are shown for $b=2, r=4$ and $c=\sqrt{2}$. One of the trajectories enters a period-2 limit cycle while the other evolves to a period-4 limit cycle. The distribution of the limit cycles of different periods in phase space is detailed in the next Section. 


\section{Attractors and basins of attraction for $b=2$}

In this Section we study at depth the structure of the attracting segment of the VGH system at the critical point $b=2$. To facilitate the analysis we express the VHG map for $b=2$ in terms of the new variable $w \equiv z-1$ as

$$
W(w)= \begin{cases}w+2, & w \leq \xi-1 \\ -w, & w>\xi-1\end{cases}
$$

One advantage of this form is that the segment of limit cycles, $\mathcal{A} \equiv[-|\xi|-$ $1,|\xi|+1]$, is symmetric in phase space with respect to $w=0$.

\subsection{Structure of the attractor}

We start our study by distinguishing the cases of positive and negative $\xi$.

\subsection{1. $\xi \leq 0$}

This case presents the simplest dynamics. The segment $\mathcal{A}$ is composed of infinite period-2 limit cycles around $w=0$, which is a fixed point.

\subsection{2. $\xi>0$}

The dynamics is more complicated for positive $\xi$. All integer initial conditions $w_{0} \in \mathbb{Z}$ lead to limit cycles with integer elements. In particular, if the initial condition is even (resp. odd), the final limit cycle will have as its elements all even (resp. odd) integers inside the segment $\mathcal{A}$. The periods of these cycles, which depend on $\xi$, are detailed in Table 1. If $w_{0} \notin \mathbb{Z}$ we need to study the cases $\xi \in \mathbb{N}$ and $\xi \notin \mathbb{N}$ separately:

- $\xi \notin \mathbb{N}$

For positive non-integer $\xi$ we have two infinite sets of limit cycles of periods $T=2(n+1)$ and $T=2(n+2)$ with $n=\lfloor\xi\rfloor$ (where $\lfloor\cdot\rfloor$ stands for the floor function). These cycles spread over $\mathcal{A}$ in a rather peculiar way. To make it clear we find useful to consider the finite set of points

$$
\mathcal{B}=\mathcal{A} \bigcap\left\{w=(-1)^{\alpha} \xi+(-1)^{\beta}(2 k+1), \quad k=0 \ldots n, \quad \alpha, \beta=0,1\right\}
$$

whose elements, when written in increasing order, we denote by $w^{i}, i=$ $1,2, \ldots$. Then, $\mathcal{B}$ punctuates a partition of the interval $\mathcal{A}$ in subintervals. Points in the same subinterval belong to cycles with the same 
period. Points in contiguous subintervals belong to cycles with different period.

For instance, if $0<\xi<1$ then $n=0$ and the frontiers of the subintervals are given by

$$
\mathcal{B}=\left\{w^{1}, w^{2}, w^{3}, w^{4}\right\}=\{-\xi-1, \xi-1,-\xi+1, \xi+1\} .
$$

Thus, in this case three subintervals exist inside the attractor. In Table 1 we have detailed the period of the limit cycle for initial conditions inside the attracting segment $\mathcal{A}$.

- $\xi \in \mathbb{N}$

When $\xi$ is a natural number every limit cycles has period $T=2(\xi+1)$.

All this information is contained in Figure 4 where we have plotted the structure of the attracting segment at $b=2$ as a function of $\xi$ with $\xi \in[-1,3]$. The different tones of gray stand for different periods. As an example, a particular $\xi^{*}<1$ has been chosen to illustrate the position of the frontiers given in (14). The discontinuous horizontal lines stand for cycles with initial conditions $w_{0} \in \mathbb{Z}$.

\subsection{Basins of attraction}

Given the limit cycle to which a point $w_{0}$ tends, its basin of attraction can be written as

$$
B_{w_{0}}=\left\{w \in \mathbb{R} \mid w=\left\lfloor w_{0}\right\rfloor+2 k \pm d, k \in \mathbb{Z}\right\}, \quad d=w_{0}-\left\lfloor w_{0}\right\rfloor .
$$

This structure of the basins of attraction is reflected in Figure 5 for two different values of $\xi$. In this figure for each initial condition $w_{0} \in[-3,3]$ we plot the cycle in $\mathcal{A}$ which traps it. The figure suggests a periodic structure in the horizontal direction. It reflects the partition of phase space into equivalence classes established by (15). More specifically, all even, odd and odd half-integer initial conditions constitute three equivalence classes by themselves. Any other initial condition generates its equivalence class by repeatedly adding alternatively $2 d$ and $2(1-d)$.

The combination of Table 1 and (15) allows to determine the period of the cycle to which an arbitrary point $w_{0}$ tends. For the sake of illustration, 
consider the case $\xi=1.5$ and the initial condition $w_{0}=2.7$. In this case $n=\lfloor\xi\rfloor=1$ and the frontiers inside the segment $\mathcal{A}$ are given by

$$
\mathcal{B}=\left\{w^{1}, w^{2}, w^{3}, w^{4}, w^{5}, w^{6}\right\}=\{-2.5,-1.5,-0.5,0.5,1.5,2.5\} .
$$

Since the chosen initial condition falls outside the attracting segment we will make use of (15) to determine another initial condition $w_{0}^{\prime} \in B_{w_{0}}$ leading to the same final limit cycle. Choosing $k=0$ we can readily find

$$
w_{0}^{\prime}=\left\lfloor w_{0}\right\rfloor+2 k-d=1.3 \in\left(w^{4}, w^{5}\right) .
$$

If we now take into account the classification detailed in Table 1 we can conclude that both $w_{0}$ and $w_{0}^{\prime}$ will enter a limit cycle of period 4 . It can be checked that this is in fact the case in the upper panel of Figure 5.

\section{Discussion of the results}

This work focuses on the coexistence of different periods in the set of neutrally stable limit cycles that mediates the bisecting bifurcation of the VGH map. We have proved algebraically that it occurs only for $b=2$. In principle, the same method might be used to determine the existence and position in parameter space of these bifurcations for any piecewise linear map.

The geometric explanation complements the algebraic approach and provides us with an intuitive way of interpreting the bifurcation in terms of the cobweb diagram. In particular, it allows us to understand the coexistence of different periods as the simultaneous presence of pieces co-linear with the bisectrix in different iterates of the map.

The structure of the attractor in $b=2$ has been described in detail. Given a certain value of the discontinuity parameter (either $c$ or $\xi$ ), the periods of the limit cycles and their location in phase space can readily be determined. The structure of the basins of attraction of the limit cycles has been resolved as well, allowing us to associate each initial condition to its final limit cycle.

The described bisecting bifurcation is accompanied by border collisions [4, 5, 10] as can be seen in Figure 2. In the example described in that figure, two unstable fixed points of $V^{[2]}(x)$ (for $b>2$ ) collide with discontinuities of the map when $b=2$ and cease to exist for $b<2$. An attempt to classify the phenomenology of border-collision bifurcations in one-dimensional discontinuous maps can be found in [11]. The classification is based on the 
linearization of the map around the collision point both in phase space and parameter space. However, the bisecting bifurcation presented here corresponds to one of the critical cases explicitly excluded from this classification.

In this study we have restricted our attention to the VGH map but what we have called bisecting bifurcations are present in other continuous and discontinuous piecewise smooth maps. However, to the best of our knowledge, they have very often gone unnoticed in the literature. In the continuous case, bisecting bifurcations can be observed in maps such as the skew tent map 12 or the map describing the dynamics of the boost converter [13]. In particular, in [14] a continuous piecewise smooth map introduced as a model of economic growth [15] is studied and the values of the parameters for which a bisecting bifurcation takes place are identified. Discontinuous maps candidates to show bisecting bifurcations can be found in [16, 17]. Our numerical experiments have shown this is in fact the case. Moreover, the map studied in [16] shows coexistence of different periods in the set of neutrally stable limit cycles with a structure very similar to the one described in this paper for the VGH map.

\section{Acknowledgements}

This work has been partially supported by contracts MCyT/FEDER, Spain FIS2007-60133 and MICINN (AYA2010-22111-C03-02). VBS thanks Generalitat Valenciana for financial support.

\section{References}

[1] J. Sprott, Chaos and time-series analysis, Oxford Univ Pr, 2003.

[2] E. Ott, Chaos in dynamical systems, Cambridge Univ Pr, 2002.

[3] S. Strogatz, Nonlinear dynamics and chaos: With applications to physics, biology, chemistry, and engineering, Westview Pr, 2000.

[4] M. Di Bernardo, C. Budd, A. Champneys, P. Kowalczyk, Piecewisesmooth dynamical systems: theory and applications, Springer Verlag, 2008.

[5] Z. Zhusubaliyev, E. Mosekilde, Bifurcations and chaos in piecewisesmooth dynamical systems, World Scientific Pub Co Inc, 2003. 
[6] G. Varley, G. Gradwell, M. Hassell, Insect population ecology: an analytical approach.

[7] R. May, G. Oster, Bifurcations and dynamic complexity in simple ecological models, American Naturalist 110 (974) (1976) 573-599.

[8] V. Botella-Soler, J. Oteo, J. Ros, Dynamics of a map with a power-law tail, J. Phys. A: Math. Theor. 42 (38).

[9] V. Botella-Soler, J. Castelo, J. Oteo, J. Ros, Bifurcations in the Lozi map, arXiv:1102.0034v1 [nlin.CD].

[10] H. Nusse, J. Yorke, Border-collision bifurcations for piecewise smooth one-dimensional maps, Int. J. Bifurcation Chaos 5 (1) (1995) 189-208.

[11] P. Jain, S. Banerjee, Border collision bifurcations in one-dimensional discontinuous maps, Int. J. Bifurcation Chaos 13 (11) (2003) 3341-3352.

[12] H. Nusse, J. Yorke, B. Hunt, E. Kostelich, Dynamics: numerical explorations, Springer Verlag, 1998.

[13] S. Banerjee, M. Karthik, G. Yuan, J. Yorke, Bifurcations in onedimensional piecewise smooth maps-theory and applications in switching circuits, IEEE Trans. Circuits Syst. I, Fundam. Theory Appl. 47 (3) (2000) 389 -394. doi:10.1109/81.841921.

[14] L. Gardini, I. Sushko, A. Naimzada, Growing through chaotic intervals, J Econ Theory 143 (1) (2008) 541-557.

[15] K. Matsuyama, Growing through cycles, Econometrica 67 (2) (1999) 335-347.

[16] V. Avrutin, M. Schanz, On multi-parametric bifurcations in a scalar piecewise-linear map, Nonlinearity 19 (3) (2006) 531.

[17] V. Avrutin, M. Schanz, On the fully developed bandcount adding scenario, Nonlinearity 21 (5) (2008) 1077. 
Table 1: Period of the trajectories according to the initial point $w_{0} \in[-|\xi|-1,|\xi|+1]$, with $b=2$ and $\xi>0(\xi \notin \mathbb{N})$. The description of the case $w_{0} \in \mathbb{Z}$ is also valid for $\xi \in \mathbb{N}$.

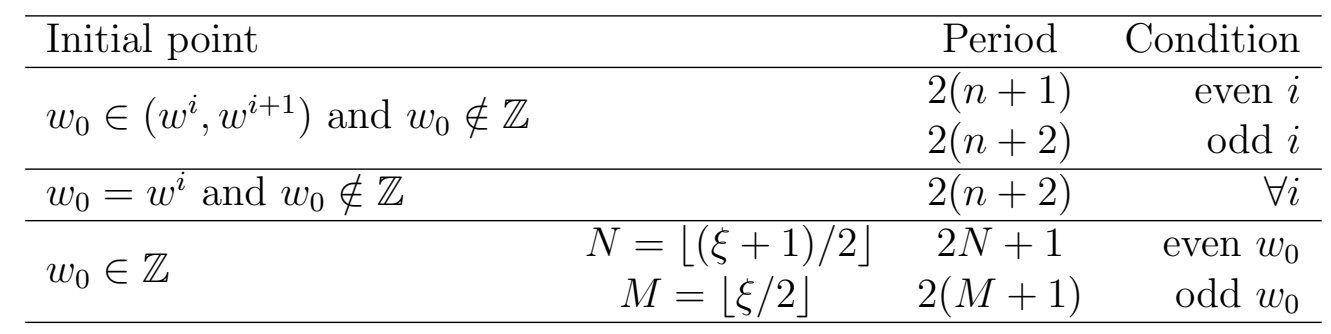

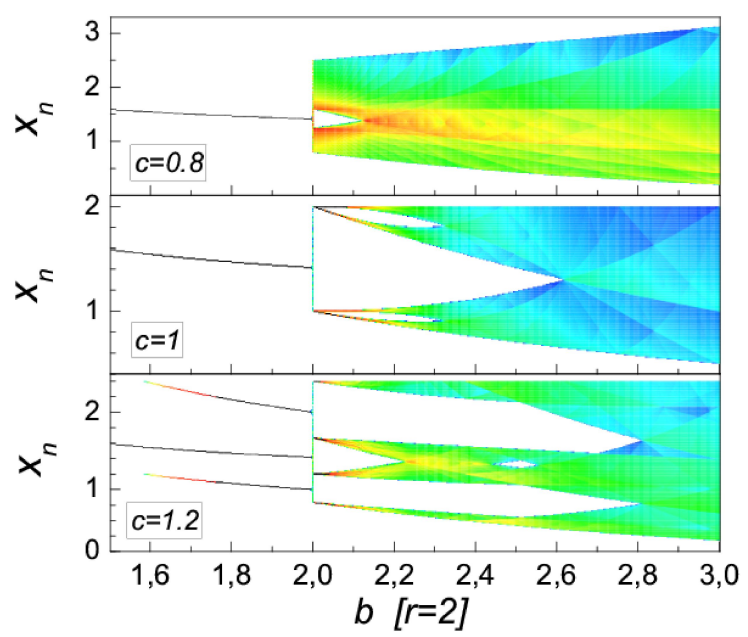

Figure 1: Bifurcation diagrams of the VGH map $V(x)$ with $r=2$ and three different values of $c$. 

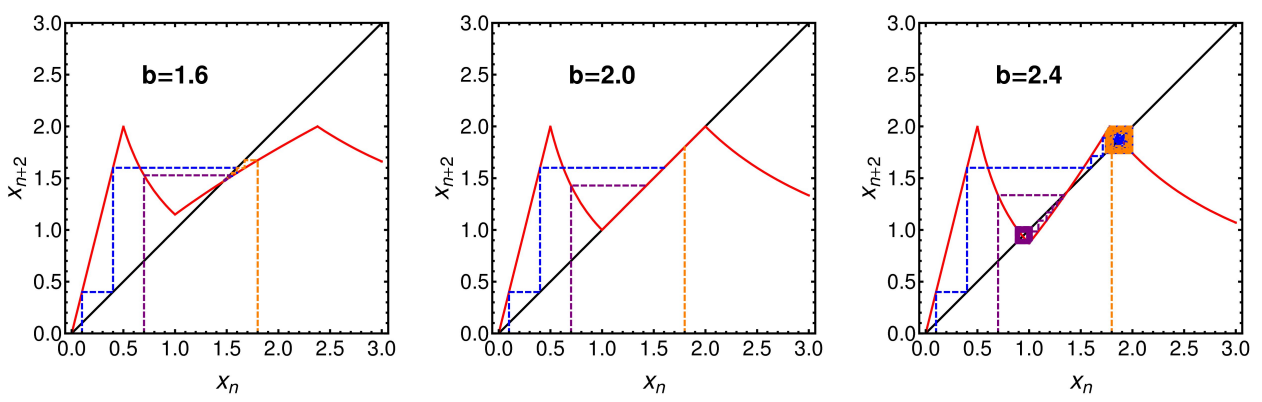

Figure 2: A sequence of three cobweb plots of $V^{[2]}(x)$ illustrating the bisecting bifurcation from order to chaos at $b=2$. Here $c=1, r=2$. The presence of the segment of fixed points is apparent in the case $b=2$.
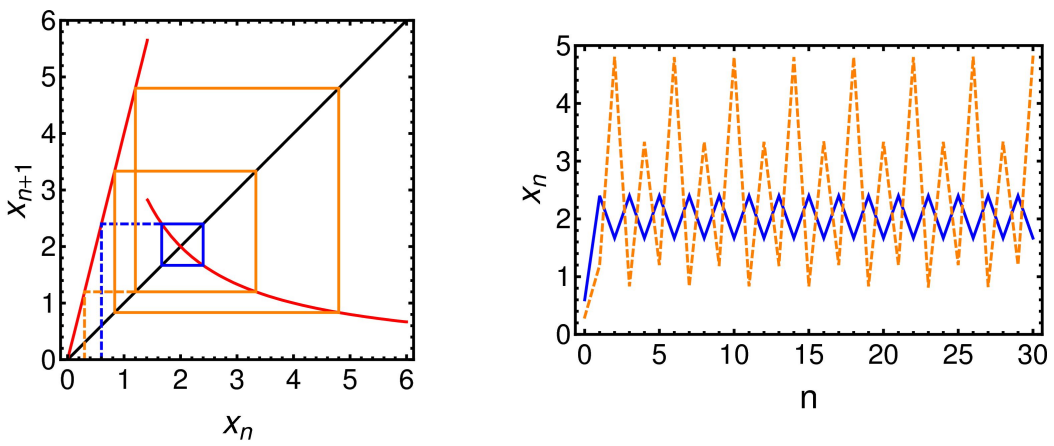

Figure 3: Cobweb diagram (left panel) and trajectories (right panel) of $V(x)$ for $b=2$, $c=\sqrt{2}, r=4$ and two different initial conditions $\left(x_{0}=0.3,0.6\right)$. It is clearly seen that each initial condition enters a neutrally stable limit cycle of different period. 


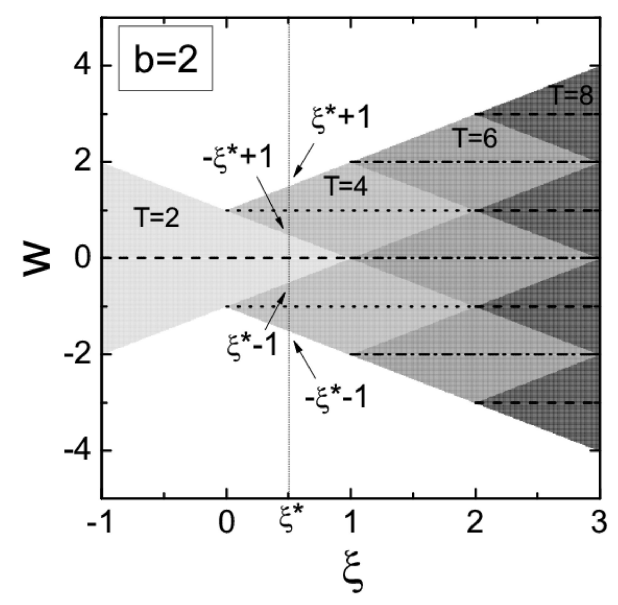

Figure 4: Structure of the attracting segment $(b=2)$ in phase space for different values of $\xi$. The different tones of gray code cycles with different periods. The discontinuous horizontal lines stand for individual cycles with integer elements of periods 1,2,3 and 4 (dashed, dotted, dash-dotted and dashed respectively) embedded in the continuum of cycles of the attracting segment.
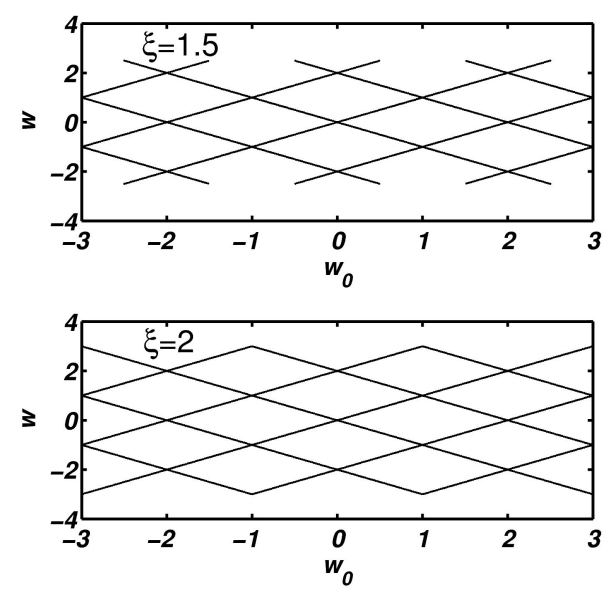

Figure 5: Structure of the basins of attraction for two different values of the discontinuity location parameter $\xi$. The graphs show the final attractor for each $w_{0}$. For $\xi=1.5$ (upper panel) infinite limit cycles of periods 4 and 6 exist as well as the period- 2 cycle $\{-1,1\}$ and the period-3 cycle $\{-2,0,2\}$ can be seen. For $\xi=2.0$ (lower panel) infinite limit cycles of period- 6 are present filling the space between the points of the period-3 cycle $\{-2,0,2\}$ and the period-4 cycle $\{-3,-1,1,3\}$. 